\title{
Reproducción asistida, gestación por sustitución y creencias. Un análisis desde las usuarias de tecnologías, los expertos religiosos y las regulaciones estatales en Argentina
}

\author{
Gabriela Irrázabal ${ }^{1}$ y María Cecilia Johnson ${ }^{2}$
}

Resumen. Este artículo se propone contribuir al análisis sobre la relación de las TRHA y las religiones, entrecruzamiento que permite poner en debate no solo los sentidos sobre la reproducción, sino también sobre la sexualidad, la familia y los sentidos culturales sobre la vida humana. Trabajaremos en tres niveles: el de las regulaciones estatales y discursos jurídicos, el de los expertos religiosos y el de las usuarias religiosas de tecnologías reproductivas. Como métodos se utilizan las entrevistas biográficas a usuarias y análisis documental de legislación, resoluciones y fallos judiciales y literatura y cursos sobre fertilidad en espacios religiosos. Ante la ampliación del uso de las TRHA, los expertos religiosos católicos intentan proponer una alternativa que se adecúe a doctrina teológica. Sin embargo, las mujeres que se consideran católicas deciden utilizar las TRHA adecuando la doctrina a sus propias convicciones. Esto se da en el marco de un proceso de individuación y autonomía y cada vez menor influencia de las instituciones religiosas en la vida privada de las personas, como señalan los principales estudios en sociales sobre religión.

Palabras clave: religión; catolicismo; tecnologías reproductivas; mujeres.

[en] Assisted Reproduction, Replacement Pregnancy and beliefs. An analysis from technology users, religious experts and state regulations in Argentina

\begin{abstract}
This article contributes to the analysis of the relationship between reproductive technologies and religions. This allows a debate on the meanings of reproduction, sexuality, the family and the cultural significances about human life. We will work on three levels: regulations and legal discourses, religious experts and religious users of reproductive technologies. As methods, biographical interviews with users and documentary analysis of legislation, resolutions and judicial decisions and literature and courses on fertility in religious spaces are used. With the extension of the use of the reproductive technologies in Argentina, the Catholic religious experts propose an alternative that adapts to theological doctrine. However, women who consider themselves Catholic, decide to use the technologies by adapting the doctrine to their own convictions. This occurs within the framework of a process of individuation and autonomy and a minor influence of religious institutions in the private life of people, as indicated by the main social studies on religion.
\end{abstract}

1 CONICET, Buenos Aires (Argentina).

E-mail: gabrielairrazabal@gmail.com

2 CONICET, Buenos Aires (Argentina).

E-mail: cecijohn27@gmail.com 
Keywords: religion; catholicism; reproductive technologies, women.

Cómo citar: Irrázabal, G. y M.C. Johnson (2019): "Reproducción asistida, gestación por sustitución y creencias. Un análisis desde las usuarias de tecnologías, los expertos religiosos y las regulaciones estatales en Argentina", Política y Sociedad, 56(2), pp. 317-339.

Sumario. 1. Introducción. 2. Materiales y métodos. 3. Regulaciones estatales y discursos jurídicos. Una cuestión de altruismo y amistad. 4. Los expertos religiosos y su propuesta de tecnología procreativa natural. 5. Usuarias religiosas de TRHA: autonomía religiosa individual y autonomía religiosa en su dimensión social. 6. A modo de cierre. 7. Bibliografía.

\section{Introducción}

Este artículo se propone contribuir al análisis sobre la relación de las TRHA y las religiones, entrecruzamiento que permite poner en debate no solo los sentidos sobre la reproducción, sino también sobre la sexualidad, la familia y los sentidos culturales sobre la vida humana en Argentina. Se aborda de manera exploratoria y descriptiva tanto la manera en que los discursos religiosos permean en el espacio público y las formas en que es regulado, así como los sentidos de mujeres religiosas que interpretan y se apropian de las tecnologías reproductivas, que suelen generar conflicto con las prescripciones sobre la reproducción basadas en las doctrinas y dogmas de sus religiones. A nivel metodológico trabajaremos desde el campo de la sociología de la religión en el marco del paradigma interpretativo de las tradiciones cualitativas. Las autoras de este artículo provienen del campo disciplinar de la sociología y el trabajo social (servicio social), disciplinas con las que se dialoga para interpretar y analizar los datos relevados. El fenómeno en estudio es complejo y, por ello, elegimos presentar los resultados de manera esquemática. Así, creemos poder, de manera sucinta, contemplar todas las dimensiones que intervienen en relación a las TRHA y las creencias en nuestro país. La utilización de las THRA en Argentina ha crecido en los últimos años debido a regulaciones recientes que universalizaron el acceso a las tecnologías (los gastos son cubiertos por los seguros de salud y el Estado). La gestación por sustitución es más limitada dado que es uno de los procedimientos que la normativa actual no contempla. Los casos que se conocen han sido autorizados por el poder judicial siempre y cuando no medie transacción económica en los procedimientos. Debido al carácter ilegal de estas prácticas se ha priorizado el análisis de fuentes documentales para poder conocer las dinámicas de estos procedimientos en la actualidad.

Se advierten procesos ambivalentes, donde las experiencias de las usuarias dan cuenta que el campo biomédico abre nuevas opciones reproductivas, pero exige procesos de autorresponsabilización y autocontrol frente a un tratamiento en ocasiones prolongado, doloroso y desconocido. Al mismo tiempo también se advierten formas de agencia en el marco de ese control, donde la religión y la espiritualidad aparecen de forma relevante en sus relatos, desde la construcción identitaria en el marco de sus biografías, como en las prácticas espirituales terapéuticas manifiestas en el espacio público, mediante devociones y rituales vinculados a sus prácticas reproductivas. De esta manera, se vislumbra en las 
usuarias diferentes relaciones e intersecciones entre la experiencia reproductiva de las prácticas religiosas y espirituales en ese marco. Los relatos manifiestan la autonomía reproductiva de las mujeres frente a la norma religiosa y una integración subjetiva de los marcos religiosos a sus propias vidas. También la religión y la espiritualidad se constituyen en un recurso que les permite a las usuarias agenciar un tratamiento complejo.

Queremos destacar que, sin desconocer los aportes de otros campos disciplinares, este artículo no comprende a las TRHA como "villanas" o "salvadoras" (Rapp, 1999); es decir, no busca ni condenarlas ni tampoco depositar en ellas la liberación del heteropatriarcado. Es por ello que no se encontrará aquí un trabajo que documente las formas de opresión de las TRHA hacia las mujeres, sino que se busca entender las diversas dimensiones religiosas y espirituales que intervienen en las experiencias reproductivas, las políticas públicas y el entramado judicial en el acceso a las tecnologías. Por ello, esquematizamos nuestro proceso de análisis en los siguientes niveles: el de las usuarias religiosas de tecnologías reproductivas, el de los discursos de los expertos religiosos y el de las regulaciones estatales sobre el acceso a las tecnologías.

Para este artículo, que es parte de una investigación mayor, se utilizan como fuente de información las entrevistas biográficas a usuarias y análisis documental de legislación, resoluciones $y$ fallos judiciales y literatura y cursos sobre reproducción de expertos religiosos. De esta manera se puede explorar las maneras en que las políticas religiosas se manifiestan en las formas de regular los cuerpos, en las normas, políticas y las decisiones de las usuarias. Antes de comenzar con el análisis de resultados, presentamos un breve estado de la cuestión con las discusiones actuales en nuestro campo disciplinar en la Argentina.

La TRHA pueden ser definidas como un conjunto de procedimientos biomédicos, tales como la inseminación artificial, la fecundación in vitro, la inyección intracitoplasmática de espermatozoides, la transferencia intrafalopiana de gametos, la transferencia de "preembriones", la transferencia a la trompa de ovocitos micro inyectados, la transferencia a la trompa de embriones en estado de pronúcleo y la clonación reproductiva; todas ellas han generado sucesivos análisis en las ciencias sociales (Ariza, 2014).

Algunas definiciones de las técnicas, como la de Ariza (2010), enfatizan la noción de voluntad de los progenitores ante la ausencia involuntaria de descendencia (2010: 3). Florencia Luna (2008) pone de relieve la posibilidad de reemplazar o colaborar en los pasos naturales de la reproducción, y en una misma línea, Naara Luna (2001) define estas técnicas a partir de la posibilidad de sustituir la reproducción de concepción, definiciones que asocian el uso de las TRHA a la llamada infertilidad.

Otro eje de discusión tiene que ver con el debate sobre la reproducción o transformación de las nociones de parentesco y familia desde las TRHA. Algunas posturas como la de Cardaci y Sánchez Bringas (2009) resultan más optimistas en este aspecto, mientras que otras posiciones destacan que, si bien la fragmentación de la idea de "maternidad" podría producir estas transformaciones de sentidos, a la vez destacan la fuerte impronta de la biologización de la familia (Hammons, 2008), donde persistiría la primacía de la familia bioconectada como ideal hegemónico (Roberts, 2008). 
Estos debates también han impactado en el campo del derecho, particularmente en relación a la filiación biológica: ¿cómo atribuir responsabilidades y derechos que devienen - hasta ahora - del parentesco? (Bergel, 2011). Las alternativas médicas y científicas ante los embriones sobrantes de las técnicas también han repercutido en el campo jurídico internacional (Lamm, 2013). La definición de la OMS, basada en la noción de infertilidad, también ha ejercido una gran influencia respecto a la manera en que se ha regulado el acceso a estas técnicas en los sistemas de salud de diferentes países. Sin embargo la misma ha sido cuestionada por su carácter heteronormativo (Farji Neer, 2015), desconociendo las experiencias de mujeres lesbianas (Ariza, 2012) así como de mujeres que eligen la monoparentalidad o "madres solteras por elección" (Jociles Rubio, 2010). A pesar de ello, en la academia estas experiencias constituyen un campo de indagación creciente que también incorpora las vivencias de mujeres gestantes en experiencias de maternidad subrogada (Berend, 2010) y, en menor medida, las experiencias de varones, a causa de su propia "infertilidad" (Galmés Belmonte, 2004; Romero Márquez, 2006).

Los desafíos políticos e ideológicos que estas técnicas han suscitado en sectores sociales y religiosos, en ocasiones antagónicos, también ha sido otro punto de discusión alrededor de estas técnicas. Desde el feminismo y los estudios de género, el debate sobre la maternidad, la reproducción y el control del sistema médico hegemónico sobre los cuerpos de las mujeres tiene un amplio recorrido. Es una de las posturas que se han posicionado críticamente frente a estas técnicas, al cuestionar la manera en que fijan lo reproductivo-maternal como parte de una (re)intervención política sobre el cuerpo de las mujeres, por la mercantilización del cuerpo de las mujeres por parte de las industrias biomédicas (Waldby y Cooper 2008), así como en la construcción de un recurso terapéutico ficticio, "la infertilidad", aun donde las tasas de efectividad son muy bajas (Ryan, 2009). La no neutralidad de estas tecnologías reproductivas en la subjetivación del feto (Petchesky, 1997) como en la fragmentación del cuerpo de la mujer (Rostagnol, 2008) constituyen otra arista que el feminismo ha cuestionado.

Otros cuestionamientos han estado asociados a las formas y posibilidades económicas para su acceso desde una política de salud (Adamson, 2009), así como aquellas posturas que desde la filosofía y la bioética analizan los desafíos que implica el descarte, la investigación, conservación de embriones y posibles programas eugenésicos (Luna, 2007).

La posición - $\mathrm{y}$ oposición - de las religiones frente a estas tecnologías constituye otra área de estudio relevante dentro de las Ciencias Sociales. Como advierte Florencia Luna (2008), el escenario es heterogéneo entre los diferentes credos, así como dentro de los mismos credos tampoco se advierten posicionamientos monolíticos. Particularmente, la Iglesia católica es el principal actor religioso que ha manifestado su oposición desde hace varias décadas (Casado, 1997; Godoy Vázquez, 2014), lo que se manifiesta tanto en diversos documentos de su doctrina como en lobby y estrategias para su obstaculización en diversos países a nivel legislativo (Vaggione, 2014). Los argumentos de la Iglesia católica aparecen expresados en un comunicado de la X Asamblea de la Academia Pontificia para la Vida (2010) titulado "La dignidad de la procreación humana y las 
tecnologías reproductivas. Aspectos antropológicos y éticos”. Academia Pontificia para la vida.

Diversas investigaciones de las Ciencias Sociales destacan la importancia de la dimensión religiosa y las creencias involucradas en las personas que se acercan a la utilización de estas técnicas. También existe un cúmulo importante de trabajos que abordan las trayectorias de mujeres y varones en relación a la reproducción asistida y las implicaciones que tiene para las relaciones familiares y de parentesco. El mandato reproductivo para las parejas heterosexuales es un denominador común en los distintos grupos religiosos que se han diferenciado en su aceptación o prohibición del uso de las tecnologías reproductivas (TRHA) total o parcialmente. Sin embargo, priman los trabajos que explican que las religiones son actores que obstaculizan o ponen límites a estas técnicas a partir del análisis de su doctrina, leyes o preceptos, al asumir que hay una relación unidireccional entre los postulados doctrinarios de las jerarquías religiosas y las actitudes y prácticas de sus fieles.

Por el contrario, se tomarán algunas posturas alternativas que permitan sortear esta limitación. Algunos estudios han enfatizado la "autonomía interpretativa" de las personas creyentes frente a las doctrinas religiosas (Dillon, 2001). Otros enfoques entienden la religión y la espiritualidad como un recurso cultural (Amermann, 2013), como investigaciones basadas en la experiencia de mujeres religiosas con las TRHA (Jennings, 2010; Czarnecki, 2012) que destacan que la religión, lejos de ser un obstáculo, contribuyó a su tránsito por el tratamiento (Olmos Álvarez, 2015). Desde perspectivas decoloniales (Mahmood, 2005) como desde la teoría performativa también se ha cuestionado el binarismo con el que la academia ha comprendido la agencia de las mujeres dentro de las religiones tradicionales, restringiéndola a las alternativas de sumisión o subversión a la norma religiosa. De esta manera proponen comprender cómo las mujeres "hacen religión" (Avishai, 2008), lo que no siempre se explica por procesos de negociación y reconciliación de identidades (Fuist, 2016).

Finalmente, más allá de la creciente autonomía de los creyentes, las religiones encuentran diferentes maneras de impactar un ámbito donde entienden se pone en juego "la creación de la vida" y que se expresa en el acceso u obstáculo al campo de la salud, como a las definiciones de quienes pueden acceder a la maternidad/paternidad.

\section{Materiales y métodos}

El presente artículo se nutre de los datos de una investigación doctoral y un proyecto de investigación financiado por el Ministerio de Ciencias y Tecnología de Argentina sobre bioética y religiones. La tesis doctoral se titula "Nuevas tecnologías reproductivas: sentidos sobre el parentesco en las trayectorias biográficas de mujeres de Córdoba" y el proyecto de investigación "Religiones y salud. La intervención. Trayectorias, representaciones y modos de intervención en el espacio público de agentes religiosos formados en bioética en Buenos Aires". Ambas investigaciones se enmarcan en la tradición de estudios cualitativos e incluyen la combinación de métodos. 
En la investigación doctoral se han realizado 17 entrevistas en profundidad a mujeres usuarias de tecnologías reproductivas entre el año 2016 y $2017^{3}$. El diseño muestral incluye mujeres de entre 35 y 65 años. Cuentan con estudios de nivel superior (terciario o universitario), profesionales de clase media-alta, con relaciones de pareja varón (13) y mujer (2), sin pareja (2). Se declaran creyentes (10), agnósticas (3) y ateas (4). Para este artículo se utilizan como insumo las entrevistas a 10 usuarias que se definieron como creyentes religiosas (apartado 5). Las entrevistas se realizaron a partir del contacto a informantes clave y con la técnica de bola de nieve.

El proyecto de investigación - aún en curso - trabaja sobre las intervenciones en el espacio público de bioeticistas religiosos. El diseño muestral está basado en la realización de entrevistas a estos expertos de grupos religiosos que participan / han participado de comisiones de bioética, el análisis documental de normativa, fallos judiciales, cursos, publicaciones y casos de bioética y la observación participante en conferencias, cursos de posgrado de bioética dictados por especialistas religiosos (hasta el momento se han realizado 12 entrevistas). La observación participante en el marco de los espacios en los que circulan los expertos nos permite captar el sentido y comprender sus acciones de manera holística. Para este artículo, hemos elegido trabajar con el análisis de dos fallos judiciales (el primero y el último dictado) de un corpus de 19 sentencias de gestación por sustitución relevadas y literatura específica sobre técnicas de reproducción asistida "natural" (Naprotechnology) en el marco del catolicismo. Se utiliza particularmente los manuales y artículos académicos sobre Naprotechnology y las conferencias dictadas por las expertas acreditadas para atender pacientes mediante el uso de esta tecnología natural (dos conferencias de junio 2016) ${ }^{4}$.

Los datos primarios del trabajo de campo (a través de entrevistas y observaciones) fueron obtenidos previo contacto, solicitud de autorización y consentimiento para las personas involucradas. En ambas investigaciones las personas fueron informadas del objetivo de los estudios que tendrían como resultado la publicación de tesis y artículos académicos. De nuestros proyectos de investigación individuales hemos seleccionado para este artículo los casos que resultan relevantes. Justificamos la decisión de analizar pocos casos por el tipo de fenómeno en estudio y porque consideramos que pueden aportar nuevos conceptos y categorías teóricas (Collier, 1993). Además, nuestro artículo no pretende generalizar, sino que pone énfasis en profundizar un fenómeno local y micro (Galeano Marín, 2004). Nuestra perspectiva epistemológica considera que los retratos, las historias, los relatos de la experiencia humana evocadores, reales, significativos, constituyen la esencia de la investigación cualitativa (Vasilachis de

\footnotetext{
En los casos recabados los tratamientos realizados han sido algunos de los que están disponibles en Argentina y los más populares. Se distiguen aquellos llamados de "baja complejidad", como la inseminación artificial (con o sin donante de esperma), y los de "alta complejidad", como la fecundación in vitro (FIV) y la inyección intracitoplasmática de espermatozoide (ICSI), los cuales pueden ser usados con gametos propios (homologos) o con gametos donados, que en los casos recabados fueron con ovodonación. No se han entrevistado aún usuarias de gestación por sustitución. En la Argentina, como es una práctica no regulada, el acceso a las personas que realizan esta modalidad ha sido dificultoso hasta el momento, por ello utilizamos los casos de la jurisprudencia porque han sido las mismas personas las que han dado a conocer públicamente su situación.

$4 \quad$ Una de las autoras de este artículo realizó observación en el año 2012 de un workshop sobre infertilidad en la Academia Pontificia para la Vida en el Vaticano, y presenció la capacitación dictada por el creador de la Naprotecnología en ese evento, el Dr. Thomas Hillgers, que le permitió tener acceso a los manuales y explicaciones sobre la técnica.
} 
Gialdino, 2006). Además, consideramos que las Humanidades y Ciencias Sociales comprenden, transmiten e interpretan discursos de otros, por ello la estrategia metodológica adoptada resulta apropiada para el objeto de estudio propuesto, ya que implica la utilización de entrevistas en profundidad y el análisis de documentos. La entrevista en profundidad, según Miguel Valles (2000), tiene las siguientes ventajas: riqueza informativa (intensiva, holística, contextualizada y personalizada), posibilidad de indagación por derroteros no previstos, flexibilidad y diligencia, y accesibilidad a información difícil de observar y preferible por su intimidad y comodidad. Asimismo, el análisis de documentos incluye la utilización de materiales textuales. Siguiendo a López Noguero (2002: 171), estudiar documentos escritos constituye un aspecto principal de investigación, ya que en las sociedades modernas la escritura ocupa una posición cada vez más destacada y predominante. A partir del análisis documental, no se pretende analizar el estilo del texto sino las ideas y el significado de las palabras o frases expresadas en él (López Noguero, 2002). Si bien es cierto que un abordaje etnográfico permitiría mezclarse con los sujetos para captar el sentido de sus acciones, hay espacios de la intimidad que nos han resultado imposibles de etnografiar (no así los espacios de intervención pública de expertos). Al margen de que el propósito del estudio no era etnografiar las prácticas reproductivas de las usuarias de las TRHA, consideramos que las entrevistas para trabajar estas temáticas son adecuadas porque ¿cómo se podría "observar" o mezclarse con los sujetos de investigación al analizar sus prácticas reproductivas si no es por medio de entrevistas y sin contravenir todos los códigos de ética de la investigación con seres humanos? Como método de recolección de datos, la entrevista tiene una larga tradición en el campo de la sociología. En palabras de Howard Becker: "Todo investigador experimentado sabe que a veces una sola entrevista es suficiente para determinar que algo es posible y esa será toda la evidencia que se necesita" (Becker, 2012: 15). A continuación comenzaremos con la descripción y análisis de resultados.

\section{Regulaciones estatales y discursos jurídicos. Una cuestión de altruismo y amistad.}

En la Argentina, se encuentra regulado el acceso a las técnicas reproductivas a partir de la ley 26862 (2013), que tiene como objeto "el acceso integral a los procedimientos y técnicas médico-asistenciales de reproducción médicamente asistida". (art. 1) $)^{5}$. Los procedimientos se realizan en todo el país en 129 establecimientos habilitados por el Ministerio de Salud (SISA, 2017). De estos centros, los nucleados en la Sociedad Argentina de Reproducción (SAMER) crearon un registro estadístico, el Registro Argentino de Fertilización Asistida. Según los últimos datos publicados, en el periodo 2004-2008, previo a la sanción

\footnotetext{
El art. 8 establece el tipo de cobertura integral: inducción de ovulación; "la estimulación ovárica controlada; el desencadenamiento de la ovulación; las técnicas de reproducción asistida (TRA); y la inseminación intrauterina, intracervical o intravaginal, con gametos del cónyuge, pareja conviviente o no, o de un donante, según los criterios que establezca la autoridad de aplicación. Quedan incluidos en el Programa Médico Obligatorio (PMO) estos procedimientos, así como los de diagnóstico, medicamentos y terapias de apoyo, con los criterios y modalidades de cobertura que establezca la autoridad de aplicación, la cual no podrá introducir requisitos o limitaciones que impliquen la exclusión debido a la orientación sexual o el estado civil de los destinatarios".
} 
de la Ley 26862, se iniciaron 14.955 ciclos, se transfirieron 12013 y se lograron 3501 embarazos clínicos (Morente, Horton, Serpa y Mc Key, 2012). Luego de la sanción de la ley, solo en el año 2015 se realizaron 9045 ciclos de alta complejidad (FIV e ICSI) con ovocitos propios, logrando 2123 embarazos clínicos (D’Agostino et al., 2017).

En el ámbito internacional, desde 1984 se registra el primer caso de gestación por sustitución, y en Argentina comienzan a aparecer casos en la jurisprudencia a partir de 2010 (Lamm, 2013). La gestación por sustitución es una práctica que quedó fuera del marco normativo, aunque no está prohibida (Scotti, 2015). Además, está la cuestión de la filiación, que está contemplada en el Código Civil, a partir de la reforma de 2015 se reconoce la "voluntad procreacional" en relación a la patria potestad (Krasnow, 2016). Al ser una práctica que no está permitida ni tampoco prohibida, los casos que llegan a la justicia se relacionan con la cuestión de la filiación, principalmente. Para la normativa argentina, la inscripción de los nacimientos está relacionada con la persona que da a luz al niño/a recién nacido mediante la certificación de una obstétrica. Los conflictos en relación a la gestación por sustitución surgen cuando las instituciones de salud emiten el certificado de nacimiento a nombre de la persona gestante. Luego de esto, los progenitores no pueden inscribir al niño/a como propio, por ello la mayoría de los casos judiciales refieren a cuestiones filiatorias. Según Rodríguez Iturburo y Notrica (2017), en Argentina hasta 2017 había 19 casos judicializados en relación a la gestación por sustitución, y consideran que al no existir legislación al respecto las personas quedan libradas a las decisiones discrecionales de los jueces. Algunos casos se presentan de forma previa a la realización de los procedimientos mediante la solicitud de autorización para la realización de la gestación por sustitución. La mayoría de las sentencias reflejan aprobaciones a los tratamientos por cuestiones de altruismo y solidaridad. Se trata de casos en los que amigas o familiares de las parejas prestan solidariamente sus úteros sin cobrar dinero a cambio.

En uno de los primeros fallos de reconocimiento de la filiación (B. M. A. c/ F. C. C. R. s/ ordinario) se consideró la cuestión de la voluntad procreacional de una pareja para realizar la inscripción del nacimiento de un niño gestado en vientre sustituto. El argumento judicial para la decisión de la autorización sintetizó las posiciones contrarias y a favor de la subrogación que circulaban en ese entonces (2013) en el país.

En contra de la gestación por sustitución según B. M. A. c/ F. C. C. R. s/ ordinario, 2013:

1) Se trata de un contrato inmoral, contrario a la dignidad de la persona.

2) Supone una explotación de la mujer debido a que conlleva la utilización de las mujeres pobres por las ricas (...). También puede conllevar situaciones de abuso respecto de el o los comitentes, generalmente "desesperados" por tener un hijo.

3) Estas prácticas podrían llevarnos a la utilización del cuerpo de la mujer como mero recinto gestador. Sin duda, esta práctica puede constituirse en una forma de manipulación del cuerpo femenino, inadmisible en una sociedad democrática.

4) La gestación por sustitución importa una cosificación de la mujer en virtud de que la gestante se convierte en un mero "ambiente" o "incubadora humana" para 
el hijo de otro. Esta cosificación, por un lado, atenta contra la libertad y autonomía de las mujeres debido a que estas no consienten libremente. Se afirma que "es discutible si las mujeres están eligiendo libremente, o si su voluntad está socialmente y económicamente influenciada". Pero, además, aunque no sea remunerado (amiga o pariente), se discute: ¿hasta qué punto las presiones familiares pueden atacar ese libre consentimiento forzando a la mujer a acceder a la gestación por sustitución?

A favor de la gestación por sustitución según B. M. A. c/ F. C. C. R. s/ ordinario, 2013:

1) Importa una manifestación del derecho a procrear. En la salvaguarda de la dignidad humana no se encuentra en absoluto el único valor fundamental que debe asegurarse frente a la gestación por sustitución, pues hay que pensar también en la protección del matrimonio o de la familia, particularmente en su tradicional función procreadora.

2) Tratándose de un acuerdo voluntario y libre, no hay por qué hablar de explotación, ni aun interviniendo dinero. El argumento de la explotación es paternalista y subestima la capacidad de consentir de la mujer.

3) Si la mujer que quiere tener un hijo precisa del óvulo de otra, sin que presente anomalías fisiológicas para gestar, aparecerá como la madre legal que pretende ser. Así, ante el análisis comparativo de las situaciones se sostiene que la admisión de la gestación por sustitución se presenta como una solución más justa. ¿Por qué es más digno y aceptable que una mujer tenga hijos con óvulos donados a que lo haga recurriendo a la donación de la "capacidad de gestación"?.

En el caso particular de la filiación, el juez recordaba que todo había sido de común acuerdo entre las partes y que la gestante solo deseaba ayudar a la familia a tener el hijo que no podía:

Nunca deseó al pequeño niño T. para sí, se siente feliz de haber ayudado a la familia a tener su tan deseado y querido hijo, es decir, que desde el primer momento decidió colaborar con la familia N. No dudó que eran ellos los que se encargarían de la crianza del niño, y tuvo en claro que ellos eran su familia, que ese era su hogar (B. M. A. c/ F. C. C. R. s/ ordinario, 2013).

Además del altruismo de la gestante, que solo habría obtenido cobertura para los gastos de salud y alimentos durante el embarazo, el juez hizo alusión a que la práctica de la gestación por sustitución se encontraba presente en el libro del Génesis del Antiguo Testamento, cuando el matrimonio de Abraham y Sara no podía concebir y tuvieron que recurrir a otra mujer (Lamm, 2013).

Una sentencia de julio de 2017 directamente autorizó a una pareja de dos hombres a transferir un embrión a una mujer. Este caso tuvo gran repercusión en la prensa nacional ya que fue considerado la "primera autorización de gestación por sustitución en la Argentina". Teniendo en cuenta los problemas para determinar la filiación, las clínicas de fertilidad comenzaron a recomendar a las parejas que 
soliciten autorización judicial de forma previa a realizar el tratamiento (Clarín, 2017).

Tanto en la sentencia como en declaraciones a la prensa, la jueza que autorizó la transferencia embrionaria basó su decisión en la consideración de la amistad de la gestante con la pareja y los motivos altruistas que la llevaron a ayudar a la pareja ("RESERVADO S/ AUTORIZACIÓN JUDICIAL (f)", Expte No 0260/17/J7).

Asimismo, incorpora al procedimiento de autorización una visita "socioambiental" a la pareja a la que declara con los medios económicos y culturales adecuados para la crianza de un hijo. Tanto la pareja como la gestante tuvieron entrevistas con peritos psicológicos del juzgado. La mujer también fue declarada con medios económicos y culturales adecuados para llevar adelante el tratamiento mediante su decisión libre e informada. ("RESERVADO S/ AUTORIZACIÓN JUDICIAL (f)", Expte No 0260/17/J7)

En medios de prensa, la jueza hizo hincapié en que el acuerdo entre las partes no incluía una retribución económica para la gestante, relató que para tomar la decisión tenía que asegurarse de que "no se tratara de un negocio" y que cuando le preguntó a la mujer, ella le dijo: “CCómo les voy a cobrar?” (Infobae, 2017). La jueza afirmó que "quería asegurarse de que no se tratara de una mujer sin recursos a la que se estuviera explotando". (Infobae, 2017).

La línea argumental que aparece en la jurisprudencia sobre gestación por sustitución destaca que los procedimientos se realizan de común acuerdo entre las partes, por amistad, sin mediar dinero y por una decisión altruista libre y autónoma de las gestantes. Cabe destacar que estos procedimientos judiciales, que han comenzado a ser recomendados por las mismas clínicas de fertilidad, incluyen la asunción por parte de la pareja del costo de los tratamientos. Le ley de fertilización asistida no incluye la gestación por sustitución como un tratamiento que cubre los seguros de salud. A continuación, veremos una propuesta de expertos religiosos católicos alternativa a la utilización de tecnologías reproductivas, a las que por doctrina teológica se oponen (Godoy Vázquez, 2014).

\section{Los expertos religiosos y su propuesta de tecnología procreativa natural}

Los discursos religiosos y en particular la Iglesia católica en América Latina han tenido una histórica injerencia sobre la sexualidad, la reproducción de sus creyentes (Pecheny y Dehesa, 2011), así como también se ha señalado su papel en la construcción de la feminidad asociada a la maternidad (Montecinos, 1990). Frente a los avances tecnológicos en el área reproductiva, que posibilitan nuevas alternativas para el acceso a la maternidad y paternidad, las religiones se han posicionado principalmente limitando y cuestionando moralmente a las usuarias y usuarios de las TRHA (Schenker, 2000), quienes se encontrarían frente a una situación dilemática frente a las enseñanzas religiosas. Los expertos religiosos, especialmente aquellos dedicados a la bioética, reconocen el legítimo deseo de los matrimonios heterosexuales a tener un hijo (Irrazábal, 2012). La función procreativa de los matrimonios es un elemento central en la teología católica. Ahora bien, cuando las parejas se ven imposibilitadas de procrear, ven en las TRHA una alternativa válida para desarrollar sus familias cristianas. Esta situación 
ha sido objeto de reflexión por las máximas autoridades de la Iglesia católica en distintos congresos y eventos científicos: ¿es posible curar la infertilidad y no sustituir con la técnica la función procreativa? (Irrazábal, 2012). Para curar la infertilidad, un médico católico norteamericano desarrolló una metodología llamada "tecnología procreativa natural" (Hillgers, 2010). Se trata de Thomas Hillgers, del Instituto Pablo VI de Omaha, EE. UU., que desarrolló un modelo de cuidado y reconocimiento de la fertilidad femenina basada en los valores de la encíclica Humanae Vitae de Pablo VI. Según Hillgers (2010), esta tecnología natural tiene mejores tasas de éxito de embarazo que las TRHA. Este método sería además sin costo para las mujeres porque solo deben aprender a conocer y liberar "el poder de su ciclo menstrual" (Hillgers, 2010). En este instituto se han formado capacitadores de esta tecnología que difunden el método en distintos países y entrenan a mujeres (y matrimonios) en el reconocimiento de los patrones de fertilidad. Incluso han desarrollado un estudio observacional con 798 participantes, el International NaProTechnology Evaluation and Surveillance of Treatment for Infertility and Miscarriage (iNEST) - ClinicalTrials.gov Identifier: NCT01363596 - con sede en la Universidad de Utah, para monitorear la tasa de éxito de esta propuesta.

El método consiste en el trabajo en conjunto con un practitioner (capacitaror / facilitador, generalmente mujer) que enseña a las mujeres a llenar una planilla en la que vuelcan los distintos estadios de su moco cervical. Con estas planillas las mujeres asisten a sus ginecólogos, quienes, sumando estudios complementarios, diagnostican las causas de la infertilidad y proponen un tratamiento (puede ser quirúrgico en caso de ser necesario). Con estas planillas a cuestas, las mujeres reconocen el funcionamiento de su ciclo y los días en que tienen posibilidad de lograr un embarazo. Una de las personas habilitadas para aplicar este método en la Argentina describió en una conferencia cómo es la enseñanza de este sistema de cuidado de la fertilidad (Richards, 2016). Se comienza por una reunión grupal en la que se presentan los principales elementos del método y el reconocimiento de la fertilidad y cómo lograr o "postergar" un embarazo.

La pareja entonces puede decidir si entra en el programa con una información detallada de lo que va a encarar y si se adapta a sus necesidades. Si decide entrar al programa, se le entrega un manual (aprox. 80 hojas), una planilla y estampas. La pareja casada deberá hacer un mes de abstinencia para no confundir los registros de moco. Se le pide que lea los primeros ocho capítulos del manual. Se les da una cita a los 15 días para su primera reunión de seguimiento. Estas reuniones personales, llamadas Follows Ups, son 8; tienen cada una, una hora - cómo mínimo- de duración. Las primeras 4 son cada 15 días, después de las cuales la pareja que ha entrado por un motivo de salud ginecológica está ya preparada para ver al médico especializado en Naprotecnología (tendrá generalmente dos ciclos registrados). Las restantes reuniones de seguimiento se distribuyen a lo largo del año. Debido a la estandarización, y a que deben conocer los significados de los biomarcadores, el sistema no puede aprenderse sin estas reuniones de seguimiento (Richards, 2016). 
Además del aprendizaje de los biomarcadores, los practitioners encaran lo que llaman el SPICE Index, referido a la relación sexual de la pareja (SpiritualPhysical-Intellectual- Creative- Emotional), en la que fomentan espacios de comunicación no basados solo en lo genital. De esta manera contribuyen a generar una relación de pareja que fomente la fertilidad (Richards, 2016). Para los expertos, el disfrute sexual de los matrimonios católicos es importante a la hora de planificar embarazos o postergarlos. Por ello realizan talleres de trabajo con las parejas en los que abordan la abstinencia genital, que no implica la abstinencia sexual (Irrazábal, 2012).

Según quienes enseñan este método en la Argentina, la tasa de eficacia ronda en el $60 \%$ en causas de infertilidad debidas a endometriosis u ovarios poliquísticos (Sánchez Ziny, 2016). En América Latina solo existen practitioners de este método en Argentina y Paraguay (Sánchez Ziny, 2016), y es un método recomendado por la Academia Pontificia para la Vida. En el ideario católico, la tecnología debe restaurar las funciones naturales, no sustituirlas (Lugo, 2016). Es decir, lo que se espera es la utilización de tecnologías reproductivas "naturales" ante la imposibilidad de procrear (matrimonios + Dios, según la teología) por cuestiones de salud. Los expertos religiosos muestran comprensión ante el deseo de los matrimonios de tener un hijo y muestran en línea con las posiciones doctrinarias (Godoy Vázquez, 2014) que no existe el "derecho a tener un hijo". Según Páez (2012), "esta actitud lleva a perder de vista que el hijo también es una persona, con su dignidad propia. Afirmar el derecho al hijo presupone considerarlo como una cosa, un objeto material del cual somos propietarios".

Las posiciones de la doctrina católica, contrarias a la utilización de las tecnologías reproductivas porque asumirían la manufactura de niños como productos carentes de defectos (Benedicto XVI, 2012) y su difusión a través de expertos en el campo de la salud y la bioética, colisionan con la experiencia vivida por las mujeres religiosas. Estas ven en las TRHA una forma de lograr sus embarazos deseados $\mathrm{y}$, al mismo tiempo, recurren a la religión como soporte espiritual para atravesar los tratamientos. A continuación, veremos estas situaciones.

\section{Usuarias religiosas de TRHA: autonomía religiosa individual y autonomía religiosa en su dimensión social}

El estudio de las experiencias de mujeres usuarias de TRHA que se reconocen como religiosas o espirituales aporta complejidad en el análisis de las decisiones reproductivas que se encuentran atravesadas por mandatos patriarcales, sociales, y religiosos. En relación a la dimensión religiosa, estas decisiones dan cuenta de constantes superposiciones entre lo que se entiende por "religioso" o "espiritual" y también incluye aquellos denominados marcos éticos y morales, que no siempre es posible discriminar de los primeros.

Mediante la comprensión de que la relación de las usuarias con la religión no se presenta de forma unidireccional, analizaremos cómo la religión y la espiritualidad forman parte de las experiencias de las mujeres usuarias con TRHA, y se advierte más bien solapamientos entre estas categorías, como señala Nancy Ammerman 
(2013). Como señalan Montenegro y Renold (2007), los límites entre lo que se entiende como "religioso" y "espiritual" son cada vez más permeables y fluidos. Se considera la religión como un "hacer" - doing religion frame- (Orit Avishai, 2008; Fuist, 2016) en el marco de una construcción identitaria que se encuentra en movimiento.

Asimismo, desde el campo de la salud, estos frecuentes entrecruzamientos entre religión y espiritualidad en el marco de diversos tratamientos suelen denominarse como tratamientos "holísticos", donde profesionales de la salud recomiendan meditación, ofrecen reiki, o donde las personas religiosas practican yoga o biodecodificación (Cornejo Valle, Blázquez Rodríguez y Flores Martos, 2014) sin resultar ello un conflicto de identidades. Aparecen ambas categorías en los relatos de las usuarias, tanto por sus posicionamientos frente a su identificación religiosa, como ante el desafío de sostener tratamientos que en ocasiones son descritos por las usuarias como "prolongados", "costosos" o "dolorosos".

Para adentrarse a los sentidos sobre la religión y la espiritualidad en el marco del tratamiento con TRHA, se analizarán diferentes dimensiones de estas categorías, tanto desde lo individual, como desde el aspecto social.

Englobamos el análisis de estas experiencias en una categoría central a la que llamamos individuación, autonomía y reflexividad de las creyentes, siguiendo a las principales teorías sobre las producciones religiosas de la modernidad (Mallimaci y Giménez Béliveau, 2007). Así también tomaremos las experiencias que reflejan la dimensión social y pública de la religiosidad en el proceso del tratamiento con TRHA, que se desarrollará en un siguiente apartado.

\section{Individuación, autonomía y reflexividad}

La pérdida de autoridad de las religiones sobre las áreas de la sexualidad y la reproducción, como los crecientes procesos de individualización, autonomía y reflexividad de las personas respecto a las normas religiosas (Mallimaci y Jiménez Béliveau, 2007; Luna, F., 2008; Giménez Béliveau e Irrazábal, 2008; Leal Reyes et al. 2011; Vaggione, 2014), permiten comprender de forma global cómo la posición - y oposición - de la religión de pertenencia sobre las TRHA no ha sido un obstáculo a la hora de iniciar los tratamientos de la casi totalidad de las usuarias entrevistadas. Las mujeres entrevistadas se reconocen como católicas y, atendiendo a la diversidad de creyentes católicos que existen en la Argentina (Giménez Béliveau e Irrazábal, 2008), podemos clasificarlas según su tipo de relación con los postulados doctrinarios en materia de sexualidad de la Iglesia católica en librepensadoras y secularizadas - más alejadas de estos dogmas - e institucionales - cercanas a estos dogmas y con autonomía de pensamiento- (Giménez Béliveau e Irrazábal, 2008). Utilizamos estas categorías analíticas como tipos ideales (Sánchez de Puerta Trujillo, 2006). Son herramientas conceptuales que como tales no existen en la realidad, sino que son construidas por los investigadores para poder comprender el fenómeno en estudio. Así, según la construcción tipológica utilizada, las secularizadas y librepensadoras practican la religión católica y sus ritos, aunque sin congregarse con frecuencia en parroquias. Las institucionales tienen mayor vida parroquial y estudios de religión, y ejercen también su libertad de pensamiento con respecto a las doctrinas oficiales (Giménez Béliveau e 
Irrazábal, 2008). En el caso de las mujeres que hemos denominado institucionales, para acceder a los tratamientos de fertilidad tuvieron que desprenderse de lo que habían aprendido sobre la reproducción en los marcos religiosos. La individuación y la autonomía se expresan en afirmaciones como tomo "mis propias decisiones" o "creo en Dios, pero no en la Iglesia" sumado a la determinación por lograr un embarazo como parte de sus planes de vida: "tenía que hacer un tratamiento", comentaban las mujeres.

Esta autonomía adquiere la forma de una reflexión ética — sopesando daños, beneficios- en sus relatos. Para Leticia (48 años, católica), lo que primó fue su propia decisión, la cual puede diferenciarse de la postura de la Iglesia católica, sin sentirse fuera de la institución por no acordar con ella en este aspecto:

Ah, pero no... a mí, la verdad lo que diga la Iglesia católica no me importa [en relación a la TRHA], yo soy católica pero católica en el sentido de que puedo ir a misa, este, puedo hablar con un sacerdote, he hecho bautizar a mis hijos, [mi hija] ha hecho la comunión, pero no, tampoco es que, o sea, tengo una mirada muy, este, no sé, o sea, no estoy de acuerdo con muchas cosas... (Leticia, 48 años).

Así también Sandra, quien se autoidentifica como católica, enfatiza nuevamente el aspecto ético y secular a la hora de decidirse por estos tratamientos, que tiene que ver con la propia valoración de "hacer bien o mal". En el caso de Catalina (38 años), quien también se autodefine como católica, fue el uso de la ovodonación el aspecto que la llevó a reflexionar sobre las posiciones de la religión católica. Al igual que Leticia, lo que primó en su decisión fue su conciencia y su autonomía para decidir sobre su cuerpo, pero siempre desde un discurso ético, de discernimiento personal, que no se encuentra opuesto a su creencia. Más aún, ella señala la heterogeneidad que existe en el campo religioso católico, y es consciente de la multiplicidad de interpretaciones de la doctrina:

Y yo soy, creo en Dios, y todo, pero creo que la Iglesia es una institución hecha por hombres y comete ochocientos errores la Iglesia. (...) La Iglesia tampoco tiene la verdad absoluta (Catalina, 38).

Otro aspecto con el que se vincula esta pérdida de centralidad de la religión y la espiritualidad en la sexualidad y reproducción de las mujeres, es la gran determinación que acompaña a la mayoría de las entrevistadas en el logro de un embarazo, lo que se relata como un fuerte pragmatismo a la hora de asumir estos tratamientos.

Entonces yo creo que hay gente que ni la piensa, va. Yo la... para mí es así es intrínseco, así como tengo que estudiar una carrera, cosas, mandatos, y además la quería, pero decir que yo tenía que adoptar o hacer un tratamiento nunca fue "iche hacemos un tratamiento?" No. Era no podemos y sí, vayamos a consultar un médico, o sea, los dos somos en ese sentido cero rollo (Catalina, 38 años).

Incluso para aquellas entrevistadas para quienes este proceso de tratamiento implicó cuestionarse la centralidad de la maternidad, aparece como una 
característica de las usuarias este llamado "pragmatismo" vinculado a cierto "sentido práctico" (Bourdieu, 1997) en sus prácticas reproductivas. Ello permitiría comprender las maneras en que se negocian los dilemas morales y religiosos frente a estas técnicas, y en la mayoría de los casos se manifiestan con una gran autonomía de la norma religiosa.

Para las entrevistadas que nosotras denominamos institucionalizadas, principalmente en aquellas con un vínculo prolongado con instituciones confesionales (por experiencias personales y laborales dentro de la Iglesia católica) seguir la propia conciencia implicó un proceso interpretativo de las propias doctrinas y enseñanzas religiosas, que Michele Dillon (2001) denomina "autonomía interpretativa" de las personas creyentes en las religiones. Ello involucró transformaciones de sentido sobre la sexualidad, la reproducción y la familia, así como sobre los sentidos de religiosidad en las biografías de las usuarias, de alguna manera en el nivel de la experiencia es posible advertir prácticas de resistencia micropolíticas (De Lauretis, 1989) a los sentidos hegemónicos de reproducción y familia.

María (43 años), quien relata una extensa socialización católica en su familia, así como una gran proximidad y formación en la Iglesia católica, si bien conocía con detalle las objeciones religiosas sobre las TRHA, ello no implicó ni un total rechazo a las enseñanzas de la religión ni una total conformidad, y señala que le "llevo tiempo tomar la decisión", a diferencia de otras entrevistadas más pragmáticas. El relato de María, que sí tuvo en cuenta la posición de la Iglesia, también da cuenta de estos procesos de reflexividad creciente dentro de las religiones que le permitieron nuevas interpretaciones sobre la fe, así como nuevas formas de habitar la religión, desde revisar los dogmas de la Iglesia católica hasta pedir consejo a un sacerdote con una mirada más flexible.

\section{La autonomía religiosa y su dimensión pública y social}

En otros relatos, la autonomía religiosa de las mujeres usuarias no tenía tanta relación con la autonomía de los dogmas religiosos, sino que se manifestaba en la posibilidad y apertura para combinar el tratamiento médico con prácticas religiosas espirituales públicas, en ocasiones como parte de un conjunto de recursos sociales vinculados a la fertilidad y la maternidad. En estos casos, la dimensión de la autonomía aparece expresada de diferentes maneras por las usuarias y puede resumirse en la siguiente afirmación: "El discurso de la religión/Iglesia sobre las TRHA no me interesa, pero las prácticas religiosas y espirituales han sido importantes durante el tratamiento". Este tipo de razonamiento, que aparece con gran frecuencia, coincide con lo señalado por las perspectivas teóricas que enfatizan el papel que tiene la religión y la espiritualidad como practica cultural, como plantea Nancy Ammerman (2013). Siguiendo a Jennings (2010) y Czarnescki (2015), si bien los discursos religiosos incrementan el peso de la reproducción sobre las mujeres, también proveen diferentes recursos culturales que permiten resistir las dificultades financieras y emocionales que experimentan durante el tratamiento.

Algunas mujeres recurrieron durante el tratamiento a prácticas vinculadas a creencias New Age y espiritualidades orientales, tales como la biodecodificación o 
las constelaciones familiares, que implica la asistencia a grupos y espacios terapéuticos, así como prácticas individuales.

En América Latina, particularmente, la dimensión pública y comunitaria de la sanación aparece frecuentemente en los relatos de las personas que atraviesan procesos de salud y enfermedad (Olmos Álvarez, 2015) vinculado a los saberes y religiosidad popular. La devoción a un santo, el rezo a la Virgen en sus diversas denominaciones, la visita a curas sanadores y misas de sanación forman parte de un itinerario terapéutico que incluye rituales religiosos, los cuales colaborarían en el éxito de los tratamientos de fertilización (Olmos Álvarez, 2015). Esos rituales terapéuticos también se manifiestan en los tratamientos con TRHA, donde coexisten con el acceso a técnicas reproductivas de alta complejidad en mujeres que no se consideran apegadas al dogma religioso como en aquellas que son creyentes más institucionalizadas. ${ }^{6}$ A su vez, esta continuidad entre saberes médicos y religiosos se advierte no solo en personas creyentes, sino que forma parte de prácticas institucionales, como ha sido señalado en algunas investigaciones, donde la "asistencia de Dios y de la ciencia" es reconocida por médicos y pacientes a lo largo de los tratamientos (Roberts, 2012; Olmos Álvarez, 2015).

Las usuarias que clasificamos como librepensadoras y secularizadas (Giménez Béliveau e Irrazábal, 2008) destacaban que a partir de su tratamiento con TRHA comenzaron a acercarse a la "religiosidad popular" o se constituyeron en una posibilidad y un recurso más durante el proceso con TRHA. Juliana (36) cuenta que, si bien se identifica como creyente católica desde niña, fue durante el proceso de búsqueda del embarazo que se acercó a los espacios de devoción, animada por una compañera de trabajo:

La virgencita, sí, yo siempre he sido devota de la Virgen de San Nicolás, pero bueno, cuando la buscaba a ella, ya no me quedaba más a donde ir, a cuál Virgen, iba a todas, iba a todas. Al último que fui, que le pedí mucho, fue al cura Brochero, y fue cuando me enteré justamente en Mina Clavero que estaba embarazada (Juliana, 36 años).

En el caso de las creyentes más institucionalizadas la religiosidad popular aparece como parte de una tradición familiar. Tal es el relato de María (43), quien, si bien se define como creyente católica, luego también señala que se define a sí misma como una "creyente sincrética", donde la tradición de sanación transmitida por su familia forma parte de su identidad católica como de los recursos con los que cuenta.

Por otra parte, esta dimensión pública de la creencia también se conecta con la manera en que se socializan los procesos reproductivos, en particular ante las dificultades para el logro de un embarazo. De esta manera la religiosidad es como un recurso de las personas próximas para acompañar el proceso de tratamiento, pero al mismo tiempo es vivido como una intervención en las decisiones reproductivas de las mujeres. Así surge del relato de Gisela:

Cabe señalar también que este tipo de tradiciones religiosas son saberes extendidos pero denostados por la institucionalidad religiosa y expresan una particular historia colonial latinoamericana, así como procesos de resistencias de los pueblos originarios. 
Bueno, mi cuñada me iba a visitar, rezaban (...). Me iba a visitar y como que no preguntaban, porque no habíamos dicho que estábamos haciendo la fertilización porque bueno, no sabíamos, pero "mandale besos a la Gise, mandale, hoy me vinieron a traer esta estampita, que era la estampita de la Virgen de la Candelaria" y le empecé a rezar a la Virgen de la Candelaria" (Gisela, 36 años).

La simbología de la Virgen de la Candelaria refiere a un tipo de denominación que está culturalmente asociada a la maternidad y la fertilidad. En este sentido el regalar una estampita a una pareja sin hijos implica una señal muy clara sobre lo deseado o esperado para esta pareja. De esta manera los objetos religiosos de la religiosidad popular a la vez que un apoyo emocional también funcionan como un mensaje sobre lo esperado y deseado para las mujeres, y pueden constituir un vehículo que puede ser instrumentalizado para reforzar los estereotipos femeninos ligados a la maternidad.

\section{A modo de cierre}

En la Argentina el uso de las TRHA es cada vez más frecuente. A partir de la ley que permite acceder a las tecnologías (a ciertas técnicas) a través de los seguros de salud y en los hospitales públicos, se amplió el panorama de derechos reproductivos. Queda aún pendiente la regulación de la gestación por sustitución que, como no está prohibida, se va regulando a través del mercado de las clínicas de fertilidad y de las presentaciones judiciales individuales. Al analizar la jurisprudencia se observa que los principales argumentos para la autorización de, en un principio, la filiación de hijos nacidos en vientre subrogado o, más actualmente, las transferencias embrionarias aluden a contratos entre amigos o familiares en los que no media intercambio comercial. Al analizar solo estas situaciones nuestro artículo presenta limitaciones, conocemos que en Argentina hay agencias comerciales de gestación por sustitución (Mannino, 2015), pero que, como son ilegales, es difícil acceder para realizar un estudio sociológico.

Ante la ampliación del uso de las TRHA, los expertos religiosos católicos, que han hecho lobby para impedir la sanción de la ley (Irrazábal, 2012), intentan proponer una alternativa que se adecúe a la doctrina teológica. Sin embargo, las mujeres que se consideran católicas deciden utilizar las TRHA adecuando la doctrina a sus propias convicciones. Esto se da en el marco de un proceso de individuación y autonomía y cada vez menor influencia de las instituciones religiosas en la vida privada de las personas, como señalan los principales estudios en sociales sobre religión.

El cruce de las experiencias de las mujeres usuarias de las TRHA con lo religioso ha permitido analizar algunos aspectos que de otro modo aparecen explicados como paradojas o contradicciones. Esto nos permite observar, por un lado, la complejidad de la experiencia de lo religioso y espiritual en nuestras sociedades actuales y, por el otro, su centralidad a la hora de analizar las decisiones sobre la sexualidad, la reproducción y la familia. 
En este sentido, con los datos propuestos hemos intentando contribuir a los debates en el campo de la sexualidad, reproducción y religión ilustrando que ante el uso de las TRHA, se observa autonomía frente a la religión tanto en los casos de gestación por sustitución como en las usuarias mujeres creyentes que no la descartan de sus marcos de sentido en sus procesos biográficos. Particularmente, las experiencias asociadas a la reproducción y la maternidad revelan fuertes interconexiones con la religión en la cultura latinoamericana. Incluso, como mencionamos, una sentencia judicial recurre a la imagen de Sara y Abraham del Antiguo Testamento para justificar que Dios estaría de acuerdo en esta práctica.

Finalmente poder analizar los diversos entrecruzamientos entre la religiosidad y el tratamiento reproductivo permite conocer las tensiones aún presentes entre los procesos secularizadores y reflexivos del proyecto moderno, pero que no se pueden desconectar de procesos sociales más amplios y de vinculaciones en la cultura religiosa latinoamericana donde persisten fuertes solapamientos entre los sentidos de familia, reproducción y religiosidad, manifiesto tanto en las formas de configuración religiosa y espiritual a nivel individual como social.

De esta manera, encontramos que aun en un campo biomédico tecnologizado y en apariencia secular, las formas de creer no se pueden desconectar de las formas de sanar, donde la categoría "salud" no puede desconocer las cosmovisiones y los sentidos que tanto expertos como usuarios le asignan a estos tratamientos.

\section{Bibliografía}

Adamson, G. (2009): “Global Cultural and Socioeconomic Factors that Inluence Access to Assisted Reproductive Technologies”, Women's Health, 5 (4), pp. 351-358.

dx.doi.org/10.2217/whe.09.28

[Consulta: 30 de marzo de 2018].

Ammerman, N. T. (2013): "Spiritual But Not Religious? Beyond Binary", Journal for the Scientific Study of Religion, 52(2), pp. 258-278.

Ariza, L. (2010): "La procreación como evento natural o tecnológico: repertorios decisorios acerca del recurso a la reproducción asistida en mujeres en parejas infértiles de Buenos Aires", EäJournal, 2 (1). Disponible en:

www.ea-journal.com/art2.1/La-procreacioncomo-evento-natural-o-tecnologico.pdf [Consulta: 30 de marzo de 2018].

Ariza, L. (2014): "La construcción narrativa de la infertilidad. Mujeres que narran la experiencia de no poder concebir", Sexualidad, Salud y Sociedad. Revista Latinoamericana, 18, pp. 41-73.

Ariza, L. (2015): "Keeping Up Appearances in the Argentine Fertility Clinic. Making Kinship Visible through Race in Donor Conception”, Tecnoscienza 6(1), pp. 5-31.

Avishai, O. (2008): "Doing Religion In a Secular World: Women in Conservative Religions and the Question of Agency", Gender \& Society, 22(4), pp. 409-433. Disponible en: http://doi.org/10.1177/0891243208321019

Becker, H. (2012): "How many qualitative interviews is enough?”, en Baker, S, y R. Edwards, Expert voices and early career reflections on sampling and cases in qualitative research, Southampton, NCRM. 
Benedicto XVI (2012): "Discurso del Santo Padre Benedicto XVI a los participantes en la Asamblea de la Pontificia Academia para la Vida", Asamblea de la Pontificia Academia para la Vida, Vaticano, 25 de febrero de 2012. Disponible en:

https://w2.vatican.va/content/benedict-xvi/es/speeches/2012/february/documents/

hf_ben-xvi_spe_20120225_acdlife.html

[Consulta: 29 de marzo de 2018 ].

Berend, Z. (2010): "Surrogate Losses: Understanding of Pregnancy Loss and Assisted Reproduction among Surrogate Mothers", Medical Anthropology Quarterly, 24 (2), pp. 240-262.

Blázquez-Rodríguez, M. (2005): “Aproximación a la antropología de la reproducción”, AIBR, Revista de Antropología Iberoamericana, (42), pp.1-25.

B. M. A. c/ F. C. C. R. s/ ordinario(2013).

Casado, M. (1997): "Reproducción humana asistida: los problemas que suscita desde la bioética y el derecho", Papers. Revista de Sociología, 53 (1), pp. 37-44, http://dx.doi.org/10.5565/rev/papers.1893.

Clarín (2017, 18 de enero) "Alquiler de vientre, embarazo y fertilidad" (en línea). Disponible en:

https://www.clarin.com/entremujeres/hogar-y-familia/embarazo/alquiler-vientreembarazo-fertilidad_0_Sy5vwJnLg.html

[Consulta: 28 de marzo de 2018].

Collier, D. (1993): "Método comparativo", Revista Uruguaya de Ciencia Política, (1) 1, pp. 21-46

Czarnecki, D. (2012): "Moral Women, Immoral Technologies: How Devout Women Negotiate Maternal Desires, Religion, and Assisted Reproductive Technologies", Gender \& Society, 29(5), pp. 716-742. http://doi.org/10.1177/0891243215591504

D’Agostino, A., F. Parera-Déniz, M. S. Robles, A. Pené, M. E. McKey, C. Morente, G. Estofán \& M. Hernández (2017): "Guía hacia la transferencia electiva de un embrión. Análisis de datos del Registro Argentino de Fertilización Asistida", Reproducción 32 (32), pp. 48-53. Disponible en:

http://www.samer.org.ar/revista/numeros/2017/ numero_4/50_55_SAEC_trabajo_original.pdf

[Consulta: 29 de marzo de 2018].

Dillon, M. (2001): "Pierre Bourdieu, Religion and Cultural Production", Cultural Studies $\leftrightarrow$ Critical Methodologies, 1(4), pp. 411-429. https://doi.org/10.1177/153270860100100402

Farji Neer, A. (2015): “Cuerpo, derechos y salud integral: Análisis de los debates parlamentarios de las leyes de Identidad de Género y Fertilización Asistida (Argentina, 2011-2013)", Salud Colectiva, 11(3), pp. 351-365.

Fonseca, C. (2008): "Homoparentalidade: novas luzes sobre o parentesco", Revista Estudos Feministas, 16(3), pp. 769-783 http://doi.org/10.1590/S0104-026X2008000300003

Fuist, T. N. (2016): "It Just Always Seemed Like it Wasn't a Big Deal, Yet I Know for Some People They Really Struggle with It": LGBT Religious Identities in Context", Journal for the Scientific Study of Religion, 55(4), pp. 770-786. http://doi.org/10.1111/jssr.12291 
Galeano Marín, M. E. (2004): Diseño de proyectos de investigación cualitativa, Medellín, EAFIT.

Garay, R. (2008): "El destino de ser madres: la ideología de a maternidad como soporte discursivo de las nuevas tecnologías reproductivas", en M. Tarducci, ed., Maternidades en el siglo XXI, Buenos Aires, Espacio, pp. 29-59.

Galmés Belmonte, I. (2004): "Utilidad y necesidad del andrólogo en las unidades de reproducción asistida", Actas Urológicas Españolas, 28 (5), pp. 364-376. dx.doi.org/10.4321/S0210-48062004000500005

Giménez Béliveau, V. y G. Irrazábal (2008): "Católicos en Argentina: hacia una interpretación de su diversidad”, Sociedad y Religión, (32-33) 32-33, pp. 442-59.

Godoy Vázquez, M. O. (2014): "Principios de los derechos confesionales sobre tecnología reproductiva e incidencia de la reproducción artificial en el régimen jurídico del matrimonio y la familia de estos ordenamientos", Foro. Nueva época, 17 (1), pp. 217-271.

Hammons, Stacy, A. (2008): “Assisted Reproductive Technologies: Changing Conceptions of Motherhood?", Afilia Journal of Women and Social Work, 23 (3), pp. 270-280.

Hillgers, T. (2010): The Naprotechnology Revolution. Unleashing the Power in a Women's cycle, Nueva York, Beaufort.

Infobae (2017): "Habla la jueza que permitió que una amiga de una pareja gay geste sus embriones" (en línea). Disponible en:

https://www.infobae.com/sociedad/2017/07/10/habla-la-jueza-que-permitio-que-unaamiga-de-una-pareja-gay-geste-sus-embriones-entendio-que-va-a-tener-un-bebe-quenunca-va-a-ser-su-hijo/

[Consulta: 28 de marzo 2018]

Irrazábal, G. (2012): Bioética y catolicismo. Estrategias e intervenciones públicas desde la bioética personalista en la Argentina (1999-2012), Tesis doctoral inédita, Universidad de Buenos Aires, Buenos Aires.

Jennings, P. K. (2010): "God Had Something Else in Mind: Family, Religion, and Infertility”, Journal of Contemporary Ethnography, 39(2), pp. 215-237. http://doi.org/10.1177/0891241609342432

Jociles Rubio, M. I. y A. M. Rivas Rivas (2010): “Motivaciones genéticas y experienciales: el discurso de las MSPE sobre la fecundación asistida como vía de acceso a la maternidad en solitario", Alteridades, 20 (39), pp. 107-124.

Krasnow, A. (2016): "Filiación por técnicas de reproducción humana asistida, gestación por sustitución y consentimiento informado en Argentina: aportes y cambios introducidos por el Código Civil y Comercial", Revista de Bioética y Derecho, 37 (37), pp. 69-84. https://dx.doi.org/10.1344/rbd2016.37.16151

Lauretis, T. D. (1989): "La tecnología del género", Technologies of Gender.

Essays on Theory, Film and Fiction, London, Macmillan Press, pp. 1-30. Disponible en: http://blogs.fad.unam.mx/asignatura/adriana_raggi/wp-content/uploads/2013/12/ teconologias-del-genero-teresa-de-lauretis.pdf [Consulta: 28 de marzo 2018]

Lamm, E. (2013): Gestación por sustitución. Ni maternidad subrogada ni alquiler de vientres, Barcelona, Universidad de Barcelona.

Lugo, E. (2016): "Pilares bioéticos, ciencia, técnica y fecundación natural", ponencia presentada en XVI Jornadas de Bioética, Comisión de Bioética P. José Kentenich, 25 de junio de 2016, Buenos Aires. 
Luna, F. (2001): "Reproducción asistida y sabor local: contexto y mujer en Latinoamérica", Revista Bioética, 9 (2), pp. 83-98.

Luna, F. (2008): Reproducción asistida, género y derechos humanos en América Latina, San José, Instituto Interamericano de Derechos Humanos.

Luna, N. (2001): "Pessoa e parentesco nas novas tecnologías reprodutivas", Revista Estudos Feministas, 9(2), pp. 389-413. http://doi.org/10.1590/S0104-026X2001000200005

López Noguero, F. (2002) "El análisis de contenido como método de investigación”, XXI Revista de Educación, 4 (4), pp. 167-180

Mahmood, S. (2005): Politics of Piety: The Islamic Revival and the Feminist Subject, Nueva Jersey, Princeton University Press.

Mallimaci, F. y V. Giménez Béliveau (2007): "Creencias e increencia en el Cono Sur de América. Entre la religiosidad difusa, la pluralización del campo religioso y las relaciones con lo público y lo político", Revista Argentina de Sociología, 5(9), pp. 44-63.

Mannino, P. (2015): "Investigan a una agencia por alquiler de vientres en Mendoza", $L a$ nación. Disponible en:

https://www.lanacion.com.ar/1821112-investigan-a-una-agencia-por-el-alquiler-devientres-en-mendoza.

[Consulta: 28 de marzo 2018]

Ministerio de Salud de la Nación- SISA (2017): Listado de establecimientos de reproducción asistida, (en línea). Disponible en:

https://www.argentina.gob.ar/sites/default/files/listado-estab.fert_.asistida-15-122017.pdf

[Consulta 27 de marzo 2018]

Montecinos, S. (1990): "Símbolo Mariano y constitución de la identidad femenina en Chile”, Estudios Públicos, 39 (39), pp, 283-290.

Montenegro, S. y J. M. Renold (2007): El fenómeno religioso: diversidad y vigencia de la $f e$, Capital Intelectual.

Morente, C., M. Horton, I. Serpa \& M. Mc Key (2012): "Registro Argentino de Fertilización Asistida (RAFA)", Reproducción, 27 (6): 6-18. Disponible en: http://www.samer.org.ar/revista/numeros/2012/vol27_n1/2.2actualidad.pdf [Consulta: 17 de Febrero 2018]

Olmos Álvarez, A. L. (2015): “Venid a mí todos los afligidos'. Salud, enfermedad y rituales de sanación en el movimiento católico carismático del Padre Ignacio", Ciencias Sociales y Religión, 17 (22), (en línea). Disponible en: http://www.seer.ufrgs.br/CienciasSociaiseReligiao/article/view/54690 [Consulta: 28 de marzo 2018]

Páez, G. (2012): "Distintas posturas sobre la valoración ética de la inseminación”, Persona y Bioética 16 (2) 137-148.

Pecheny, M. y R. D. Dehesa (2011): "Sexualidades y políticas en América Latina: un esbozo para la discusión”, (en línea). Disponible en: http://www.sxpolitics.org/pt/wp-content/uploads/2009/10/sexualidades-y-politicas-enamerica-latina-rafael-de-la-dehesa-y-mario-pecheny.pdf [Consulta: 30 de marzo 2018]

Petchesky, R. P. (1987): "Fetal Images: The Power of Visual Culture in the Politics of Reproduction", Feminist Studies, 13(2), pp. 263-292. http://doi.org/10.2307/3177802 
Rapp, R. (1999): Testing Women, Testing the Fetus: The Social Impact of Amniocentesis in America (Anthropology of Everyday Life), Routledge.

Roberts, E. S. (2012): God's laboratory: assisted reproduction in the Andes, Berkeley, University of California Press.

Reservado s/ autorización judicial (f), Expte No 0260/17/J7 (2017) Disponible en: https://dpicuantico.com/sitio/wp-content/uploads/2017/07/Jurisprudencia-Familia14.07.2017.pdf

[Consulta: 28 de marzo 2018]

Richards, B. (2016): "Presentación de situaciones de infertilidad y asistencia interdisciplinaria", ponencia presentada en XVI Jornadas de Bioética, Comisión de Bioética P. José Kentenich, 25 de junio de 2016, Buenos Aires. Disponible en: www.familia.org.ar [Consulta: 29 de marzo 2018]

Roberts, E. S. (2012): God's laboratory: assisted reproduction in the Andes, Berkeley, University of California Press, 2012.

Rodríguez Iturburo, M. y F. Notrica (2017): “La figura de la gestación por sustitución: otra vez en boca de todxs", Microjuris, (en línea). Disponible en:

https://aldiaargentina.microjuris.com/2017/09/15/la-figura-de-la-gestacion-porsustitucion-otra-vez-en-boca-de-todxs-rodriguez-iturburu-mariana-notrica-federico-p/ [Consulta: 29 de marzo 2018]

Romero Márquez, N. R. (2002): "Representación social de las técnicas de reproducción asistida", Revista Facultad de Medicina, 25 (2), pp. 231-236.

Rose, N. (2007): The Politics of life itself. Biomedicine, Power and Subjetivity in the Twenty First Century, Nueva Jersey, Princeton University Press.

Rostagnol, S. (2008): “El papel de 1@s ginecólog@s en la construcción de los derechos sexuales en Uruguay", en K. Araujo y M. Prieto, eds, Estudios sobre sexualidades en América Latina, Quita, FLACSO, pp. 215-232.

Ryan, M. A. (2009): "The Introduction of Assisted Reproductive Technologies in the 'DevelopingWorld': A Test Case for Evolving Methodologies in Feminist Bioethics", Signs, 34 (4) pp. 805-825.

Salem, T. (1997). “As novas tecnologías reprodutivas: o estatuto do embrião e a noção de pessoa", Mana, 3(1), pp. 75-94. http://dx.doi.org/10.1590/S0104-93131997000100003

Sánchez de Puerta Trujillo, F. (2006) "Los tipos ideales en la práctica: significados, construcciones, aplicaciones", Empiria, Revista de Metodología de Ciencias Sociales, 11 (11), pp. 11-32

Sánchez Ziny, G. (2016): “Tecnología procreativa natural. Na pro tecnología, el cuidado de la fertilidad", ponencia presentada en XVI Jornadas de Bioética, Comisión de Bioética P. José Kentenich, 25 de junio de 2016, Buenos Aires.

Schenker, J. G. (2000): "Women's reproductive health: Monotheistic religious perspectives", International Journal of Gynecology and Obstetrics, 70(1), pp. 77-86. http://doi.org/10.1016/S0020-7292(00)00225-3

Scotti, L. (2015): "La maternidad subrogada en la legislación y jurisprudencia argentinas", (en línea). Disponible en: http://docs.wixstatic.com/ugd/05acff_abb18e381754402191325cd639c85ef1.pdf [Consulta: 29 de marzo 2018] 
Vaggione, J. M. (2014): "La politización de la sexualidad y los sentidos de lo religioso", Sociedad y Religión, 42(42), pp. 209-226.

Cornejo Valle, M., M. Blázquez Rodríguez y J. A. Flores Martos (2014): "El incómodo vínculo entre medicina y fe", en M. Cornejo Valle, M. Blázquez Rodríguez y J. A. Flores Martos (comps.), El reencuentro de salud y espiritualidad: agencias, saberes y prácticas, Actas del XIII Congreso Nacional de Antropología. Periferias, fronteras y diálogos, 4485-4492, Tarragona, Universitat Rovira i Virgili.

Valles, M. (2000): Técnicas cualitativas de investigación social, Madrid, Síntesis.

Vasilachis de Gialdino, I. (2006): Estrategias de investigación cualitativa, Barcelona, Gedisa.

Waldby, C. y M. Cooper (2008): "The Biopolitics of Reproduction: Post-Fordist Biotechnology and Women's Clinical Labour", Australian Feminist Studies, 23(55), pp. 57-73.

http://doi.org/10.1080/08164640701816223 